01,11

\title{
Спиновые флуктуации и концентрационные магнитные переходы в киральных геликоидальных ферромагнетиках $\mathrm{Fe}_{1-x} \mathrm{Co}_{x} \mathrm{Si}$
}

\author{
(C) А.А. Повзнер, А.Г. Волков, Т.А. Ноговицына, С.А. Бессонов \\ Уральский федеральный университет, \\ Екатеринбург, Россия \\ E-mail: a.a.povzner@urfu.ru
}

Поступила в Редакцию 19 августа 2019 г.

В окончательной редакции 19 августа 2019 г.

Принята к публикации 3 сентября 2019 г.

\begin{abstract}
Флуктуационная теория применяется к исследованию концентрационных превращений в киральных геликоидальных ферромагнитных квазибинарных неупорядоченных сплавах $\mathrm{Fe}_{1-x} \mathrm{Co}_{x} \mathrm{Si}$ со взаимодействием Дзялошинского-Мория. Основное состояние описывается на основе используемых в ab initio pacчетах приближений $\mathrm{LDA}+\mathrm{U}+\mathrm{SO}$, с дополнительным учетом концентрационных флуктуаций, связанных с различием потенциалов внутриатомного хаббардовского взаимодействия на узлах, занятых атомами железа и кобальта. Рассматриваются решения полученных уравнений магнитного состояния для фаз дальнего и ближнего порядков с правой и левой магнитной киральностью. Исследуются концентрационные зависимости параметров межмодового взаимодействия и области составов, в которых имеют место индуцированные тепловыми флуктуациями магнитные фазовые переходы первого рода, сопровождаемые возникновением флуктуаций спиновой спирали. Показано, что переход с изменением знака магнитной киральности сопровождается возникновением минимума на концентрационной зависимости параметра мода-мода и возникновением квантового геликоидального ферромагнетизма с заметным усилением нулевых спиновых флуктуаций.
\end{abstract}

Ключевые слова: геликоидальный ферромагнетизм, спиновые флуктуации, электронная структура.

DOI: 10.21883/FTT.2020.01.48737.569

\section{1. Введение}

Сильно коррелированные геликоидальные ферромагнетики $\mathrm{Fe}_{1-x} \mathrm{Co}_{x} \mathrm{Si}$ являются прототипами современных спинтронных материалов, однако природа формирования в них не тривиальных и неоднородных спиновых магнитных фаз недостаточно изучена. Рассматриваемая группа сплавов относится к структурному типу В20 с пространственной группой $P 2{ }_{1} 3$, для которой характерно отсутствие центра инверсии [1], вследствие чего возникает антисимметричное релятивистское обменное взаимодействие Дзялошинского-Мория (ДМ) с фиксированной магнитной киральностью. Конкуренция ДМ-взаимодействия с неоднородным обменным взаимодействием приводит к формированию длиннопериодических геликоидальных спиновых спиралей с аномально большими (по сравнению с другими киральными геликоидальными ферромагнетиками со структурой В20) магнитными периодами, порядка 100-1000 § [2]. Причины таких повышенных значений магнитных периодов, так же как их аномально резкое концентрационное возрастание вблизи концентрации $x_{C}=0.65$, при которой наблюдается переход в не геликоидальное состояние с параметром ДМ-взаимодействия, равным нулю [3], требуют дальнейших исследований.

Известно [4], что причиной фазовых переходов первого рода, сопровождаемых возникновением неустойчивых ферромагнитных состояний с флуктуациями спирали и формированием скирмионных фаз является смена знаков параметров межмодовой связи в функционале Гинзбурга-Ландау. Поэтому термодинамический анализ устойчивости возможных спиновых фаз необходимо дополнить исследованиями особенностей DOS в окрестности уровня Ферми, что возможно в рамках первопринципных исследований электронной структуры.

До настоящего времени результаты таких расчетов в применении к магнитным сплавам системы $\mathrm{Fe}_{1-x} \mathrm{Co}_{x} \mathrm{Si}$ остаются явно недостаточными для исследования концентрационных зависимостей магнитных свойств. $A b$ initio $\mathrm{LSDA}+\mathrm{U}+\mathrm{SO}$-расчеты магнитных моментов основного ферромагнитного состояния $\mathrm{Fe}_{1-x} \mathrm{Co}_{x} \mathrm{Si}$ приводят к расчетным значениям, которые заметно превышают экспериментальные данные (см. $[5,6]$ и ниже рис. 3). В работе [6] исследование концентрационных зависимостей магнитных моментов было выполнено в GGA + U схеме виртуального кристалла, учитывающей неоднородное пространственное распределение спиновой плотности (градиентное приближение). Хотя при таком подходе согласие с экспериментальными данными значительно улучшилось, однако не удалось получить удовлетворительное согласие с наблюдаемыми концентрационными границами магнитоупорядоченной области, что, по-видимому, указывает на преувеличение роли пространственных неоднородностей, описываемых градиентными поправками. 
Поправки к приближению виртуального кристалла также рассматривались в рамках спин-флуктуационной модели с учетом LSDA $+\mathrm{U}+\mathrm{SO}$-электронных спектров для составов с $x=0.3$ и 0.5 [7]. Однако не были получены уравнения для локальной намагниченности, решения, описывающие смену знака магнитной киральности $\left(x=x_{C}\right)$, а также не исследовались возможные эффекты нулевых спиновых флуктуаций (возможность квантовой критичности?). Не рассматривались причины того, почему неустойчивости ферромагнитной профазы, приводящие к подавлению геликоидального дальнего порядка и возникновению ближнего порядка с флуктуациями спирали и скирмионными решетками, возникают только в интервале концентраций $x$ от 0.2 до 0.5 [2].

B настоящей работе, на основе DOS, полученных путем $a b$ initio LDA + U-зонных расчетов, проведенных для широкой области концентраций, исследуются полученные уравнения магнитного состояния, определяющие концентрационно-температурную зависимость локальной намагниченности, неоднородные спиновые фазы, спиновые флуктуации, возникающие при температурноконцентрационных переходах. Поправки, связанные с концентрационными флуктуациями, рассматриваются самосогласованно, а не через локальную намагниченность, определяемую в схеме LSDA + U + SO, как это было сделано в [7]. Рассматриваются причины возникновения концентрационных областей, в которых при фазовых переходах первого рода формируются фазы с флуктуациями спиновой спирали, концентрационного перехода при $x=x_{C}$ с изменением знака спиновой киральности. Получено, что при $x>x_{C}$ формируется область квантового геликоидального ферромагнетизма.

\section{2. Модель ферромагнитной профазы}

Рассмотрим сильно коррелированную электронную систему киральных магнетиков $\mathrm{Fe}_{1-x} \mathrm{Co}_{x} \mathrm{Si}$ с гамильтонианом, учитывающим энергию зонного движения, внутриатомные кулоновские спиновые и зарядовые корреляции, с учетом различия внутриатомных и кулоновских взаимодействий на узлах, занятых атомами $\mathrm{Fe}$ на Со. Исходя из $a b$ initio расчетов электронной структуры сплавов $\mathrm{Fe}_{1-x} \mathrm{Co}_{x} \mathrm{Si}$, отметим, что уровень Ферми находится в верхней энергетической зоне, сформированной преимущественно $t_{0}$-состояниями, в которой орбитальным вырождением и хундовским взаимодействием можно пренебречь. Гамильтониан модели Хаббарда-Канамори [8] в таком случае будет выглядеть следующим образом:

$$
\mathscr{H}=H_{0}+\delta \mathscr{H}_{\text {int }},
$$

где $H_{0}=\sum_{\mathbf{k}, \sigma} \varepsilon_{\mathbf{k}, \sigma} a_{\mathbf{k}, \sigma}^{+} a_{\mathbf{k}, \sigma}-$ гамильтониан зонного движения сильно коррелированных $d$-электронов в $t_{0}$-орбитальном состоянии, $a_{\mathbf{k}, \sigma}^{+}\left(a_{\mathbf{k}, \sigma}\right)-$ оператор рождения (уничтожения) электрона в $t_{0}$-зоне, $\mathbf{k}-$ вектор квазиимпульса, $\sigma(= \pm 1)-$ спиновый индекс, $\varepsilon_{\mathbf{k}}^{(\mathrm{LDA})}-$ электронный спектр $d$-электронов в $t_{0}$-орбитальном состоянии, рассчитанный в $\mathrm{LDA}+\mathrm{U}+\mathrm{SO}-$ приближении и приближении виртуального кристалла и отсчитываемый от энергии химического потенциала рассматриваемой электронной системы.

$$
\begin{aligned}
\delta \mathscr{H}_{\mathrm{int}}= & \left(U_{\mathrm{Fe}}-U_{\mathrm{Co}}\right) \sum_{\boldsymbol{v}} \delta p_{\boldsymbol{v}} \sum_{\sigma}\left\langle n_{\boldsymbol{\sigma}}\right\rangle_{0} \delta n_{\boldsymbol{v}} / 2 \\
& -\sum_{\boldsymbol{v}}\left(U_{\mathrm{Fe}}\left(1-p_{\boldsymbol{v}}\right)+U_{\mathrm{Co}} p_{\boldsymbol{v}}\right)\left[\left(S_{\boldsymbol{v}}^{(z)}\right)-\left(\delta n_{\boldsymbol{v}}\right)^{2} / 4\right]
\end{aligned}
$$

- поправка, включающая в себя флуктуации электронной плотности, которые обусловлены межэлектронными корреляциями и различием параметров хаббардовского взаимодействия на узлах, оккупированных атомами кобальта или железа $\left(U_{\mathrm{Co}}\right.$ и $U_{\mathrm{Fe}}-$ coответственно), $\delta p_{v}=p_{v}-p, p-$ концентрация атомов кобальта, $p_{v}$ - проекционный оператор, который может принимать значения 0 на узле, занятом железом, и 1 , если узел занят кобальтом, $\left(p_{v}^{2}=p_{v}\right)$, $n_{\boldsymbol{v}, \sigma}=a_{\boldsymbol{v}, \sigma}^{+} a_{\boldsymbol{v}, \sigma}, S_{\boldsymbol{v}}^{(z)}=\sum_{\boldsymbol{\sigma}} \sigma n_{\boldsymbol{v}, \sigma} / 2, \delta n_{\boldsymbol{v}}=n_{\boldsymbol{v}}-\sum_{\boldsymbol{\sigma}}\left\langle n_{\boldsymbol{\sigma}}\right\rangle_{0}$, $n_{. v}=\sum_{\sigma} n_{v, \sigma},\left\langle n_{\boldsymbol{v}, \sigma}\right\rangle_{0}=\left\langle n_{\sigma}\right\rangle_{0}-$ числа заполнения спиновых $d$-состояний на узле в приближениях LDA + U и виртуального кристалла.

Для записи статистической суммы перейдем в мацубаровское представление взаимодействия и введем единичные по модулю векторы $\mathbf{e}_{v}$, которые в момент мацубаровского „времени“ $\tau$ направлены вдоль оси квантования оператора спинана узле $\boldsymbol{v}-\mathbf{S}_{\boldsymbol{v}}=S_{\boldsymbol{v}}^{(z)} \mathbf{e}_{\boldsymbol{v}}$. Выполняя усреднение выражения для статистической суммы по всем возможным направлениям этих векторов, имеем

$$
\begin{aligned}
Z_{p} & =\int_{0}^{4 \pi}(d \Omega) \mathrm{Sp} T_{\tau} \\
& \times \exp \left\{-H_{0} / T+\sum_{\boldsymbol{v}}\left(U_{\mathrm{Fe}}-U_{\mathrm{Co}}\right) \delta p_{v} \sum_{\sigma}\langle n\rangle_{0} \delta n_{v} / 2\right. \\
& \left.-\sum_{\boldsymbol{v}}\left(U_{\mathrm{Fe}}\left(1-p_{\boldsymbol{v}}\right)+U_{\mathrm{Co}} p_{\boldsymbol{v}}\right)\left[\left(\mathbf{e}_{\boldsymbol{v}} \mathbf{S}_{\boldsymbol{v}}\right)^{2}-\left(\delta n_{\boldsymbol{v}}\right)^{2} / 4\right]\right\},
\end{aligned}
$$

где $(d \Omega)=\Pi_{v} d \Omega_{v}, d \Omega_{v}-$ элемент телесного угла направлений единичного вектора $\mathbf{e}_{v}, \boldsymbol{v}=(\boldsymbol{v}, \tau)$.

Далее, для того чтобы свести многочастичные взаимодействия в (2) (которые соответствуют квадратичным слагаемым по оператору спиновой плотности) к взаимодействию электронов с флуктуирующими обменными полями $(\xi)$, используем процедуру формализма преобразований Стратоновича-Хаббарда [9]

$$
\begin{aligned}
Z_{p} & =\int(d \xi d \eta)(d \Omega) \\
& \times \exp \left\{-\sum_{q}\left|\xi_{q}\right|^{2}-\sum_{q}\left|\eta_{q}\right|^{2}\right\} Z\left(\xi_{\mathbf{q}}, \rho_{\mathbf{q}},\left\langle n_{\sigma\rangle_{0}}\right),\right.
\end{aligned}
$$


где

$$
\begin{gathered}
Z(\zeta)=\operatorname{sp} T_{\tau} \exp \left(-T^{-1} H_{0}^{(\mathrm{LDA})}-T^{-1} \widetilde{\mathscr{H}}\right) \\
(d \xi d \eta)=d \xi_{0} d \eta_{0} \Pi_{q \neq 0, j=1,2} d \xi_{\mathbf{q}}^{(j)} d \eta_{\mathbf{q}}^{(j)}
\end{gathered}
$$

(индекс $j$ нумерует реальную и мнимую части стохастических $\xi$ - и $\eta$-полей),

$$
\begin{gathered}
\tilde{\mathscr{H}}=2 \sum_{q} \mathbf{S}_{q} \xi_{-\mathbf{q}}+i \sum_{q} n_{q} \rho_{-\mathbf{q}} / 2 \\
\boldsymbol{\zeta}_{-\mathbf{q}}=c\left(\xi_{-q} \mathbf{e}_{-q}+(2 U)^{-1}\left(U_{\mathrm{Co}}-U_{\mathrm{Fe}}\right) \sum_{v} \delta p_{v} e^{i q v}\right) \\
\rho_{-\mathbf{q}}=c\left(\eta_{-q}-(2 U)^{-1}\left(U_{\mathrm{Co}}-U_{\mathrm{Fe}}\right) \sum_{v} \delta p_{v} \sum_{\sigma}\left\langle n_{\sigma}\right\rangle_{0} e^{i q v} / 4\right),
\end{gathered}
$$

$\mathbf{S}_{q}$ - фурье-образы оператора вектора $\mathbf{S}_{v}, c=(U T)^{1 / 2}$, $U=(1-p) U_{\mathrm{Fe}}+p U_{\mathrm{Co}}, q=\left(\mathbf{q}, \omega_{\mathbf{2 n}}\right), \omega_{2 n}-$ мацубаровская бозе-частота.

\section{3. Уравнение магнитного состояния с учетом DM-взаимодействия}

Для того чтобы описать ферромагнитное геликоидальное упорядочение, выражение, полученное для свободной энергии ферромагнитного состояния, необходимо дополнить малой поправкой, которая описывает энергию взаимодействия Дзялошинского-Мория. Поле Дзялошинского будем вводить феноменологически, и в силу его малости ограничимся его учетом в приближении среднего поля. Выполним следующую замену

$$
H_{\mathrm{eff}} \rightarrow H_{\mathrm{eff}}-\sum_{m}\left[\mathbf{h}_{\mathbf{q}_{0}, m}^{(D)} \times \mathbf{S}_{-\mathbf{q}_{0}, m}\right]
$$

а затем

$$
\xi_{\mathbf{q}_{0}, m} \rightarrow \xi_{\mathbf{q}_{0}, m}-\mathbf{h}_{\mathbf{q}_{0}, m}^{(D)} / c,
$$

где $\mathbf{h}_{\mathbf{q}_{0}^{(D)}}=\left[\mathbf{M}_{\mathbf{q}_{-, m}} \times \mathbf{d}_{\mathbf{q}_{0}}\right]-$ среднее поле Дзялошинского, $\mathbf{d}_{\mathbf{q}_{0}}=i d \mathbf{q}_{0}, d-$ постоянная Дзялошинского-Мория, $\mathbf{M}_{\mathbf{q}_{0}, m}\left(=\left\langle\mathbf{S}_{\mathbf{q}_{0}, m}\right)-\right.$ вектор неоднородной намагниченности на векторе $\mathbf{q}_{0}$.

В рассматриваемой задаче о фазовых переходах в киральных магнетиках с аномально большими периодами магнитной структуры квантовостатистическое вычисление выражения для функционала свободной энергии $Z\left(\xi,\left\langle n_{\sigma}\right\rangle_{0}\right)$ выполним на основе приближения однородных локальных полей [10]. Разлагая (4) по степеням $\widetilde{\mathscr{H}}$ и проводя квантовостатистическое усреднение, получаем ряд по степеням внутренних обменных и зарядовых полей, действующих на электроны, $-\xi_{q}$ и $\rho_{q}$.

Вычисления функциональных интегралов выполним в приближении метода наибыстрейшего спуска по переменным: $\xi_{0}, \xi_{ \pm \mathbf{q}_{0}}^{(\gamma)}, \eta_{q},\left|\xi_{q}^{(\gamma)}\right|$ и $\phi_{q}^{(\gamma)}\left(=\arg \xi_{q}^{(\gamma)}\right)$, перевальные значения которых определяются условиями максимума подынтегрального выражения в (4). Условия перевала для зарядовых полей, согласно уравнению электронейтральности

$$
x=\sum_{\alpha} \int f(\varepsilon-\mu) g_{\alpha}(\varepsilon) d \varepsilon
$$

сводятся к виду

$$
\rho_{v}=\left(|\xi| \sum_{\alpha} g_{\alpha}(\varepsilon)\right)^{-1} \sum_{\alpha} \alpha g_{\alpha}(\varepsilon)\left(\left|\xi_{v}\right|^{2}-|\xi|^{2}\right),
$$

где $|\xi|^{2}=N_{0}^{-1} \sum_{v} \xi_{v}^{2}$. При этом плотность электронных состояний вследствие расщепления электронных термов флуктуирующими обменными полями разбивается на подзоны с плотностями состояний

$$
g_{\alpha}(\varepsilon)=g_{0}^{(\mathrm{LDA})}\left(\varepsilon+\alpha U_{m}\right)
$$

где значениям $\alpha=+1$ соответствуют низко энергетические состояния, а $\alpha=-1-$ высоко энергетические состояния.

Анализ выражения для статистической суммы (4) показывает, что имеется связь между перевальными значениями $\xi$-полей с намагниченностями $M_{0}=U^{-1}\left(c \xi_{0}^{(z)-h}\right)$ и $\mathbf{M}_{\mathbf{q}_{0}, m}=U^{-1}\left(c \boldsymbol{\xi}_{\mathbf{q}_{0}}-\mathbf{h}_{\mathbf{q}_{0}}\right)$ на векторах $\mathbf{q}=0$ и $\pm \mathbf{q}_{0}$, и среднеквадратическим магнитным моментом на узле, определяемом значениями модуля намагниченности и амплитуды спиновых флуктуаций

$$
m^{2}=(c / U)^{2} \sum_{v} \xi_{v}^{2}+U\left(U_{\mathrm{Co}}-U_{\mathrm{Fe}}\right)(c / U)^{2} \sum_{v}\left(\delta p_{v} \xi_{v}\right)^{2}
$$

Если принять условия гомеополярности и хаотического сплава $\left\langle\delta p_{\nu} \delta p_{\mu}\right\rangle=x(1-x) \delta_{v, \mu}$, получим два вклада в среднеквадратический спиновый момент, связанные с фрустрациями и квантовыми спиновыми флуктуациями, которые перенормированы концентрационными флуктуациями хаббардовского потенциала

$$
m^{2}=\left(1+x(1-x) U^{-1}\left(U_{\mathrm{Co}}-U_{\mathrm{Fe}}\right)\right)\left(\sum_{\mathbf{q}= \pm \mathbf{q}_{0}}\left|\mathbf{M}_{\mathbf{q}}\right|^{2}+\left\langle m^{2}\right\rangle\right) .
$$

Кроме того, согласно условиям седловой точки, имеем

$$
\begin{aligned}
& 2 r_{q \gamma}^{2}\left(\left\langle D^{-1}\right\rangle_{p}+a+\langle\kappa\rangle_{p} \sum_{= \pm \mathbf{q}_{0}}\left|M_{\mathbf{q}, \gamma}\right|^{2}\right. \\
& \left.\quad+\langle\kappa\rangle_{p} x(1-x) U^{-1}\left(U_{\mathrm{Co}}-U_{\mathrm{Fe}}\right) \sum_{\mathbf{q}= \pm \mathbf{q}_{0}}\left|M_{\mathbf{q}, \gamma}\right|^{2}+X_{q}\right)=1
\end{aligned}
$$

откуда

$$
\begin{aligned}
\left\langle m^{2}\right\rangle & =(T / U) \sum_{q}\left(2 r_{q}^{2}+1\right) \\
& =(2 \pi)^{-1} \sum_{\mathbf{q}\left(\neq \mathbf{q}_{0}\right)} \int_{0}^{\infty}\left(1+2 f_{B}(\omega / T)\right) \operatorname{Im}\left\langle T_{\tau} \mathbf{S}_{q, m} \mathbf{S}_{-q, m}\right\rangle d \omega .
\end{aligned}
$$


Здесь $f_{B}(\omega / T)$ - функция Бозе-Эйнштейна,

$$
\begin{aligned}
\left\langle D^{-1}\right\rangle_{p} & =1-U \chi_{\perp}(m) \\
& +\left(1+x(1-x) U^{-1}\left(U_{\mathrm{Co}}-U_{\mathrm{Fe}}\right)\right)\langle\kappa\rangle_{p}\left(\left\langle m^{2}\right\rangle\right) / 3
\end{aligned}
$$

- фактор обменного усиления однородной магнитной восприимчивости.

Коэффициент межмодовой связи

$$
\langle\kappa\rangle_{p}=\left(U / m^{2}\right)\left[\chi_{\perp}(m)-\chi_{\|}(m)\right]
$$

можно выразить через поперечную и продольную восприимчивости $\chi_{\perp}=\left(2 U m_{p}\right)^{-1} \Delta n \quad$ и $\chi_{\|}=$ $=2\left(\sum_{\alpha= \pm 1} g_{\alpha}(\mu)\right)^{-1} \Pi_{\alpha= \pm 1} g_{\alpha}(\mu)$, причем

$$
\Delta=\sum_{\alpha= \pm 1} \alpha \int_{-\infty}^{\infty} g_{\alpha}(\varepsilon) f(\varepsilon-\mu) d \varepsilon
$$

есть разность чисел заполнения низко- и высокоэнергетических состояний с $\alpha= \pm 1$ („правильные“ и „не правильные“ спины).

Наконец, из условий минимума статистической суммы (4) по однородной и неоднородной намагниченности получаем уравнения магнитного состояния для локальной и неоднородной намагниченностей

$$
\begin{aligned}
\mathbf{M}_{\mathbf{q}_{0}} & \left(\left\langle D^{-1}\right\rangle_{p}+\langle\kappa\rangle_{p} x(1-x) U^{-1}\left(U_{\mathrm{Co}}-U_{\mathrm{Fe}}\right)\right. \\
& \left.\times \sum_{\mathbf{q}- \pm \mathbf{q}_{0}}\left|\mathbf{M}_{\mathbf{q}, \gamma}\right|+X\left(\mathbf{q}_{0}, 0\right)\right)+\langle\kappa\rangle_{p} \mathbf{M}_{\mathbf{q}_{0}, 0}\left(\mathbf{M}_{\mathbf{q}_{0}}\right)^{2} \approx \mathbf{h}_{\mathbf{q}},
\end{aligned}
$$

где $\mathbf{h}_{\mathbf{q}}=\mathbf{h}, \mathbf{h}_{\mathbf{q}, \gamma}^{(D)}, \mathbf{M}_{\mathbf{q}_{0}}\left(=\mathbf{M}_{-\mathbf{q}_{0}}^{*}\right)=2^{-1 / 2}\left(\mathbf{i} M_{\mathbf{q}_{0}}^{(x)}+\mathbf{j} M_{\mathbf{q}_{0}}^{(y)}\right), \mathbf{i}$ и j - орты, лежащие в геликоидальной плоскости, $\mathbf{h}$ - вектор внешнего однородного магнитного поля в единицах магнетона Бора.

\section{4. Решения уравнения магнитного состояния}

В рассматриваемом случае неупорядоченных сплавов имеем два типа решений уравнения магнитного состояния. Когда параметр межмодового взаимодействия положителен $(\kappa>0)$ и фактор обменного усиления $D<0$, решения уравнения магнитного состояния (12) соответствуют ферромагнитному геликоиду, в котором вектор амплитуды геликоидальной структуры фиксирован. В таком магнетике возможна как левая, так и правая магнитная киральность

$$
\begin{gathered}
M_{\mathbf{q}_{0}}^{(x)}=M_{S} / 2, \quad M_{\mathbf{q}_{0}^{(y)}}= \pm i M_{S} / 2 \\
M_{S}=\left(2\langle\kappa\rangle_{p}\right)^{-1 / 2}\left(\left(\left\langle D^{-1}\right\rangle_{p}+\langle\kappa\rangle_{p} x(1-x) U^{-1}\left(U_{\mathrm{Co}}-U_{\mathrm{Fe}}\right)\right.\right. \\
\left.\left.\times \sum_{\mathbf{q}= \pm \mathbf{q}_{0}}\left|\mathbf{M}_{\mathbf{q}}\right|^{2}+X(\mathbf{q}, 0)\right)^{2}-\left(d q_{0} / U^{2}\right)\right)^{1 / 4}
\end{gathered}
$$

Последнее уравнение имеет два решения, соответствующие двум разным знакам магнитной киральности (разность квадратов в (14)).
Если знак межмодового взаимодействия отрицательный $(\kappa<0)$, то в зависимости от знака $D$ возникают флуктуации левой либо правой спирали

$$
M_{\boldsymbol{v}}^{(x)}=M_{S} \cos \left(\mathbf{q}_{0} \boldsymbol{v}+\varphi\right)
$$

и

$$
M_{v}^{(y)}=\mp M_{S} \sin \left(\mathbf{q}_{0} \boldsymbol{v}+\varphi\right),
$$

где фаза $\varphi$, из-за исчезновения ферромагнитной оси квантования, меняется стохастически.

При магнитном переходе киральность возникающих флуктуаций спирали должна быть такой же, как и киральность исходного геликоидального упорядочения.

Модуль волнового вектора геликоидального упорядочения $\mathbf{q}_{0}$ определяется условием максимума модуля вектора амплитуды неоднородной намагниченности. В модели Линдхарда для паулиевской восприимчивости

$$
\begin{aligned}
& \chi^{(0)}(\mathbf{q}, \Omega)=\chi^{(0)}(0,0)\left(1+A\left(\mathbf{q} / k_{F}\right)^{2}\right. \\
& \left.\quad-i B\left|U_{\mathbf{q}} / k_{F}\right|^{-1} \omega \theta\left(T_{0}\left|\mathbf{q} / k_{F}\right|-\omega\right)\right) \theta\left(2 k_{F}-|\mathbf{q}|\right)
\end{aligned}
$$

имеем: $\left|\mathbf{q}_{0}\right| \approx d / 2 U A$..

Аналогично [11] можно показать, что решения для флуктуаций спирали справедливы в области ферромагнитных спиновых корреляций, радиус которых описывается выражением

$$
R_{C}=k_{F}^{-1} A^{1 / 2}\left(\left|\langle\kappa\rangle_{p}\right|\left(2\left|\mathbf{M}_{\mathbf{q}_{0}}\right|^{2}+\left\langle m^{2}\right\rangle\right)\right)^{-1 / 2} .
$$

Причиной изменения знаков $D$ и $\kappa$ могут быть концентрационные и (или) температурные скачки амплитуды квантовых (например нулевых) спиновых флуктуаций. Пример таких эффектов дает магнитный фазовый переход в MnSi [11], который сопровождается скачкообразным подавлением нулевых спиновых флуктуаций и возникновением кроссовера фазового перехода первого рода и квантового перехода.

Температура исчезновения решений, описывающих геликоидальный дальний порядок, соответствует температуре максимума однородной магнитной восприимчивости, которая, согласно уравнениям (12), имеет вид

$$
\chi=2 U^{-1}\left[\left(\left|\langle\kappa\rangle_{p}\right|\left(2\left|\mathbf{M}_{\mathbf{q}_{0}}\right|^{2}+\left\langle m^{2}\right\rangle\right)-X\left(\mathbf{q}_{0}, 0\right)\right)^{-1}-1\right] .
$$

\section{5. Анализ эксперимента в модели DOS $\mathrm{Fe}_{1-x} \mathrm{Co}_{x} \mathrm{Si}$}

Для численного анализа полученных выражений рассчитана плотность электронных состояний с использованием метода LDA $+\mathrm{U}+\mathrm{SO}$. При расчетах использовался программный пакет Elk. Структурные данные для исследуемых сплавов были заимствованы из [12]. В частности, учитывалось, что постоянная решетки 

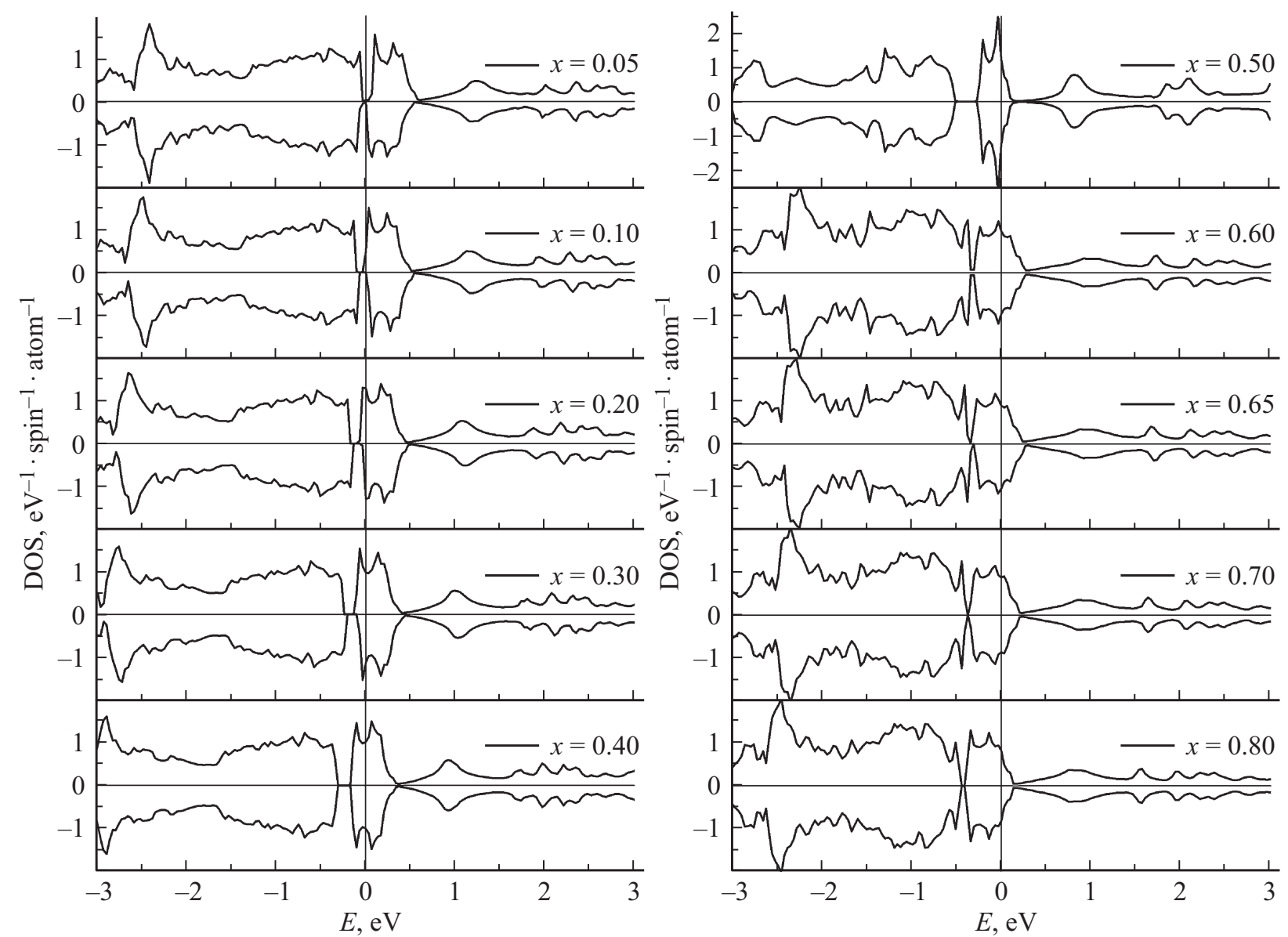

Рис. 1. Плотности электронных состояний сплавов $\mathrm{Fe}_{1-x} \mathrm{Co}_{x} \mathrm{Si}$, рассчитанные в методе LDA $+\mathrm{U}+\mathrm{SO}$. Положение химического потенциала совпадает с началом отсчета энергии. Параметры хаббардовского взаимодействия вычислялись в приближении виртуального кристалла: $U=(1-x) U_{\mathrm{Fe}}+x U_{\mathrm{Co}}, U_{\mathrm{Co}}=2.4 \mathrm{eV}, U_{\mathrm{Fe}}=1.2 \mathrm{eV}, x-$ концентрация кобальта.

изменяется линейно с ростом концентрации кобальта. При этом имеет место изменение атомной киральности исследуемых сплавов.

На рис. 1 приведены рассчитанные плотности электронных состояний для сплавов $\mathrm{Fe}_{1-x} \mathrm{Co}_{x} \mathrm{Si}$ с $x=0.05-0.8$ в модели виртуального кристалла. Видим, что плотность состояний рассматриваемых составов состоит из двух подзон, разделенных энергетической щелью. Зона, в которой находится уровень Ферми в обоих случаях, формируется преимущественно синглетными $t_{0}$-электронными состояниями. Расчеты хундовского взаимодействия для этой энергетической полосы дают пренебрежимо малые значения по сравнению с рассчитанными величинами $U$.

Далее рассчитывались амплитуды нулевых и тепловых спиновых флуктуаций в случае неупорядоченного сплава. Используя модель Линдхарда (14) и уравнение магнитного состояния (12) было получено

$$
\begin{aligned}
\left\langle m^{2}\right\rangle_{0} & =\left(4 \pi^{2} A^{2} B\right)^{-1} \sum_{\gamma}\left[\left(\left\langle D^{-1}\right\rangle_{p}+2\langle\kappa\rangle_{p} M_{S}^{2}\right)^{2}-A^{2}\right] \\
& \times\left[1+\ln \left(1+B^{-1}\left(\left\langle D^{-1}\right\rangle_{p}+2\langle\kappa\rangle_{p} M_{S}^{2}\right)^{2}\right)\right]
\end{aligned}
$$

$$
\begin{aligned}
\left\langle m^{2}\right\rangle_{T}= & (3 / 4) B(T / U)^{2}\left(\left\langle D^{-1}\right\rangle_{p}+2\langle\kappa\rangle_{p} M_{S}^{2}\right)^{-1} \\
& \times\left(\left\langle D^{-1}\right\rangle_{p}+2\langle\kappa\rangle_{p} M_{S}^{2}+A\right)^{-1}
\end{aligned}
$$

Значения параметров функции Линдхарда $A$ и $B$ определялись из сопоставления результатов расчетов магнитной восприимчивости с экспериментальными данными (рис. 2). Параметры Дзялошинского-Мория, используемые в расчетах, были заимствованы из работы [2].

Концентрационная зависимость магнитного момента сплавов $\mathrm{Fe}_{1-x} \mathrm{Co}_{x} \mathrm{Si}$ в основном состоянии приведена на рис. 3. Полученная в настоящей работе зависимость локальной намагниченности от концентрации улучшает согласие с экспериментальными данными на концентрационных границах магнитоупорядоченной области, по сравнению с результатом LDA + DMFT-расчетов [6].

Графики концентрационно-температурных зависимостей $k$ построены на рис. 4. Анализ концентрационных зависимостей $\kappa(x)$ и $D(x)$ в рассматриваемой модели $\mathrm{Fe}_{1-x} \mathrm{Co}_{x} \mathrm{Si}$ показывает, что в основном состоянии геликоидальный ферромагнетизм в интервале от $x_{1}=0.05$ до $x_{C}=0.65$ является термодинамически устойчивым, причем нулевыми флуктуациями можно пренебречь. 
При концентрации $x_{c}$ реализуется ферромагнетизм. Исчезновение геликоидального дальнего порядка при концентрации $x_{c}$ сопровождается формированием стохастических концентрационных флуктуаций связанных с возникновением волн спиновой плотности, имеющих всевозможные значения волновых векторов q (см. (14)). В области концентраций $x_{c}<x<x_{2}(=0.80)$ получаем,

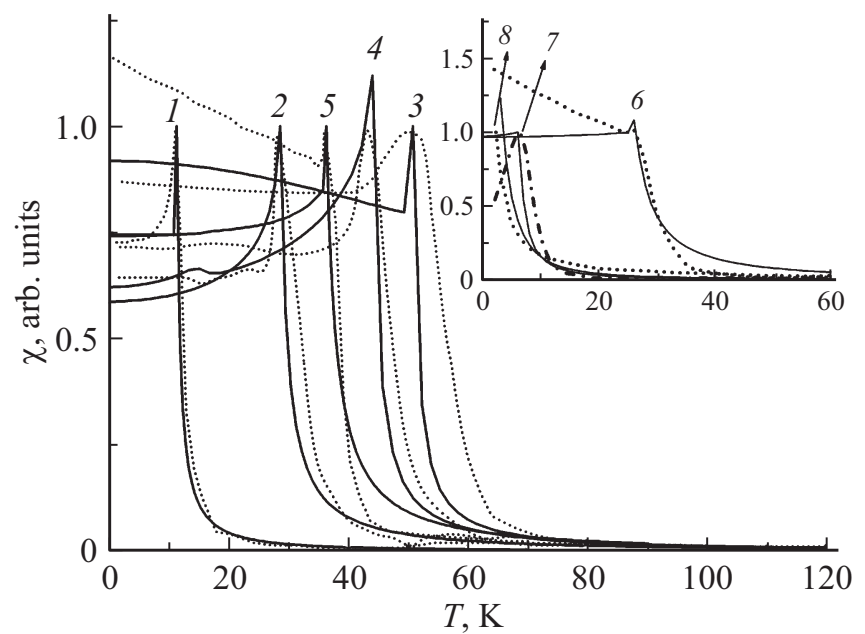

Рис. 2. Температурная зависимость магнитной восприимчивости $\mathrm{Fe}_{1-x} \mathrm{Co}_{x} \mathrm{Si}$, нормированная на максимальное значение. Пунктирная линия - экспериментальные данные [13], сплошная линия - расчет в настоящей работе: $(1)-x=0.1$; (2) $-x=0.2$; (3) $-x=0.3$; (4) $-x=0.4$; (5) $-x=0.5$; (6) $-x=0.6 ;(7)-x=0.7 ;(8)-x=0.8$. Параметр А для всех составов равен $1 / 12$, а параметр $B:(1)-3.9$; (2) - 1.3; (3) $-0.95 ;(4)-0.82 ;(5)-0.98 ;(6)-0.92 ;(7)-0.82$; $(8)$ - 0.75. Уменьшение параметра $B\left(=\left(m^{*} / m_{0}\right)^{1 / 2}\right)$ с увеличением концентрации $x$ связано с уменьшением эффективной массы электронов $\left(m^{*}\right)$ по мере удаления от запрещенной зоны.

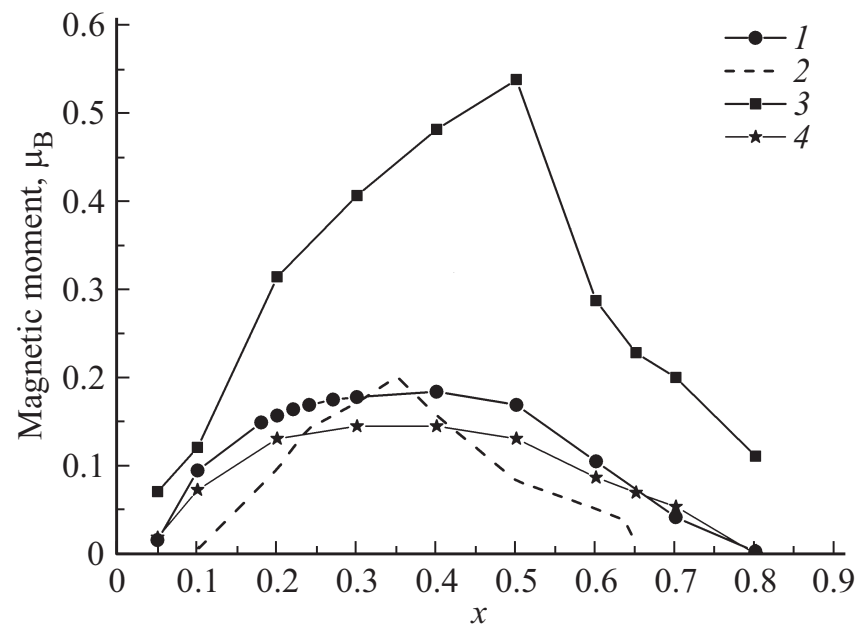

Рис. 3. Концентрационная зависимость магнитного момента сплавов $\mathrm{Fe}_{1-x} \mathrm{Co}_{x} \mathrm{Si}$ в основном состоянии: $(1)-$ экспериментальные значения [15]; (2) - расчет магнитного момента в приближении LDA + DMFT [6]; (3) - расчет магнитного момента в приближении LSDA в настоящей работе; $(4)-$ расчет в настоящей работе.

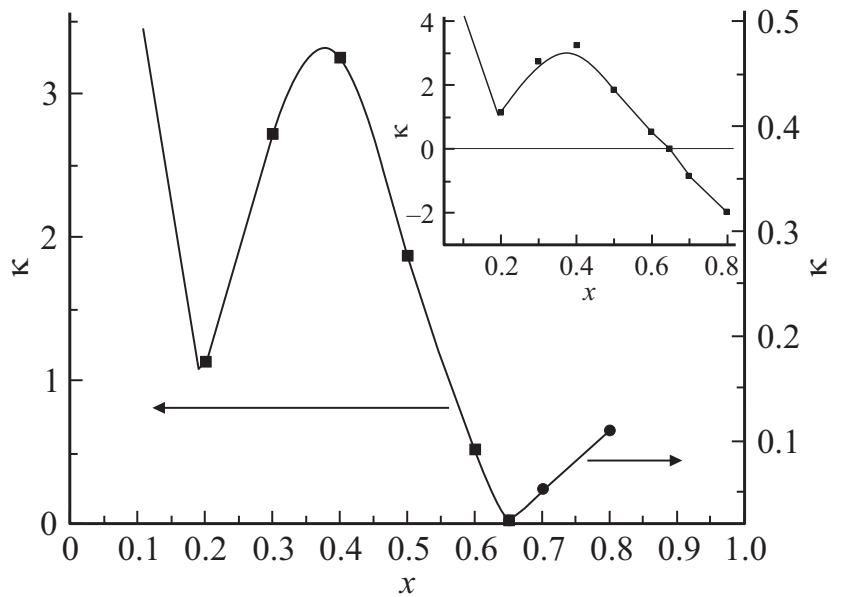

Рис. 4. Концентрационная зависимость параметра межмодового взаимодействия сплавов $\mathrm{Fe}_{1-x} \mathrm{Co}_{x} \mathrm{Si}$ в основном состоянии с учетом нулевых флуктуаций. На вставке: концентрационная зависимость параметра межмодового взаимодействия в основном состоянии без учета нулевых флуктуаций.

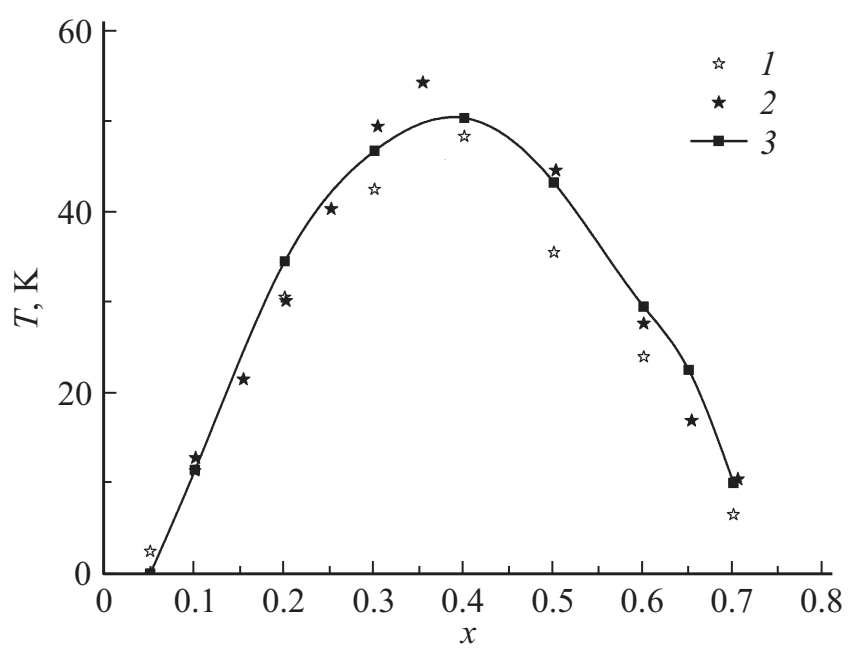

Рис. 5. Концентрационная зависимость температуры КюриНееля $\left(T_{C}\right):(1)$ - экспериментальные данные $[13] ;(2)-$ экспериментальные данные [14]; (3) - расчет в настоящей работе.

что наблюдаемое на эксперименте геликоидальное ферромагнитное состояние характеризуется правой киральностью спиновой спирали и может возникнуть только вследствие усиления нулевых спиновых флуктуаций.

Из уравнения (12) также следует, что при фазовом переходе первого рода (составы с $x=0.2-0.5)$ возникает область геликоидального ближнего порядка с ненулевой локальной намагниченностью (15). Температура исчезновения локальной намагниченности $\left(T_{s}\right)$ в этом случае не совпадает с температурой Кюри-Нееля $\left(T_{C}\right)$

$$
T_{S}^{2}=T_{C}^{2}+\left(4 U^{2} A\right)^{-1}\left(U_{\mathrm{Co}}^{1 / 2}-U_{\mathrm{Fe}}^{1 / 2}\right)^{2} d^{2} x(1-x),
$$

что и наблюдается для составов с $0.20<x<0.50$. Для остальных магнитоупорядоченных составов с геликои- 
дальным ферромагнетизмом фазовый переход в точке $T_{C}$ не является переходом первого рода и сопровождается не скачкообразным исчезновением локальной намагниченности. Концентрационная зависимость температуры $T_{c}$ приведена на рис. 5.

\section{6. Заключение}

В настоящей работе показано, что наряду с термодинамическим и квантовыми спиновыми флуктуациями в киральных ферромагнетиках на основе квазибинарных сплавов приходится рассматривать концентрационные флуктуации кулоновских потенциалов различных магнитоактивных атомов. При этом для построения самосогласованной процедуры учета таких флуктуаций следует рассматривать не LSDA + U + SO модель, а „стартовать“ от $\mathrm{LDA}+\mathrm{U}+\mathrm{SO}$ приближения для энергетического электронного спектра основного состояния. Используя такой спектр в гамильтониане Хаббарда, расширенном учетом двух типов внутриатомного кулоновского взаимодействия, можно рассмотреть основные особенности концентрационно-температурных зависимостей магнитных свойств киральных ферромагнетиков $\mathrm{Fe}_{1-x} \mathrm{Co}_{x} \mathrm{Si}$ c кристаллической структурой В20. Полученные концентрационные зависимости локальных намагниченностей улучшают согласие с экспериментальными зависимостями на концентрационных границах магнитоупорядоченной области, по сравнению с результатом LDA + DMFTрасчетов [6]. Полученные с учетом LDA + U + SO расчетов концентрационные зависимости температур переходов и температурные зависимости магнитных восприимчивостей находятся в согласии с экспериментом.

В рамках развитого подхода описаны реализуемые в данной системе дальние геликоидальные ферромагнитные порядки с разным знаком киральности, причем при концентрации, при которой такое изменение киральности происходит, имеет место изменение знака параметра межмодовой связи и реализуется область, описываемая сильным спиновым ферромагнитным ближним порядком. При „включении“ в таком ферромагнитном состоянии ДМ-взаимодействия могут возникать либо флуктуации спиновой спирали, либо, как в рассмотренном случае, геликоидальный ферромагнетизм с противоположным знаком магнитной киральности.

Рассмотрены концентрационные области, в которых имеют место температурные фазовые переходы первого рода и возможно возникновение флуктуаций спиновых спиралей. Следует ожидать [15], что во внешнем магнитном поле именно в этих концентрационных областях будут возникать скирмионные фазы. Однако в отличие от квантовых геликоидальных ферромагнетиков $\mathrm{MnSi}$, $\mathrm{Fe}_{1-x} \mathrm{Mn}_{x} \mathrm{Si}[11,17]$ возникновение таких фаз не будет сопровождаться скачками амплитуд нулевых спиновых флуктуаций. Поэтому представляет интерес сравнительный анализ тройных диаграмм магнитных состояний: температура-магнитное поле-концентрация, для $\mathrm{Fe}_{1-x} \mathrm{Co}_{x} \mathrm{Si}$ и $\mathrm{Fe}_{1-x} \mathrm{MnSi}$.

\section{Финансирование работы}

Результаты были получены в рамках задания министерства образования и науки Российской Федерации, контракт 3.9521.2017/8.9.

\section{Конфликт интересов}

Авторы заявляют, что у них нет конфликта интересов.

\section{Список литературы}

[1] J. Beille, J. Voiron, F. Towfiq, M. Roth, Z.Y. Zhang. J. Phys. F 11, 2153 (1981).

[2] С.В. Григорьев, В.А. Дядькин, С.В. Малеев, D. Menzel, J. Schoenes, D. Lamago, E.B. Москвин, H. Eckerlebe. ФTT 52, 852 (2010).

[3] S.-A. Siegfried, E.V. Altynbaev, N.M. Chubova, V. Dyadkin, D. Chernyshov, E.V. Moskvin, D. Menzel, A. Heinemann, A. Schreyer, S.V. Grigoriev. Phys. Rev. B 91, 184406 (2015).

[4] M. Janoschek, M. Garst, A. Bauer, P. Krautscheid, R. Georgii, P. Boni, C. Pfleiderer. Phys. Rev. B 87, 134407 (2013).

[5] M.P.J. Punkkinen, K. Kokko, M. Ropo, I.J. Väyrynen, L. Vitos, B. Johansson, J. Kollar. Phys. Rev. B 73, 024426 (2006).

[6] V.V. Mazurenko, A.O. Shorikov, A.V. Lukoyanov, K. Kharlov, E. Gorelov, A.I. Lichtenstein, V.I. Anisimov. Phys. Rev. B 81, 125131 (2010)

[7] А.А. Повзнер, А.Г. Волков, Т.А. Ноговицына. ФТТ 60, 227 (2018).

[8] V.I. Anisimov, J. Zaanen, O.K. Andersen. Phys. Rev. B 44, 943 (1991).

[9] J. Hubbard. Proc. Roy. Soc. A 276, 238 (1963).

[10] J.A. Hertz, M.A. Klenin. Phys. Rev. B 10, 1084 (1974).

[11] A.A. Povzner, A.G. Volkov, T.A. Nogovitsyna. Physica B Condens. Mater. 536, 408 (2018).

[12] S.V. Grigoriev, D. Chernyshov, V.A. Dyadkin, V. Dmitriev, S.V. Maleyev, E.V. Moskvin, D. Menzel, J. Schoenes, H. Eckerlebe. Phys. Rev. Lett. 102, 037204 (2009).

[13] Y. Onose, N. Takeshita, C. Terakura, H. Takagi, Y. Tokura. Phys. Rev. B 72, 224431 (2005).

[14] П.В. Гельд, А.А. Повзнер, Л.Ф. Ромашева. ДАН СССР 265, 1379 (1982).

[15] A.A. Povzner, A.G. Volkov, T.M. Nuretdinov. Solid State Commun. 298, 113640 (2019).

[16] А.А. Повзнер, А.Г. Волков, Т.М. Нуретдинов. ФТТ 61, 630 (2019).

Редактор Ю.Э. Китаев 